\title{
Population pharmacokinetic analysis of tacrolimus in Chinese cardiac transplant recipients
}

\author{
Yan Gong, ${ }^{1}$ Ming Yang, ${ }^{2}$ Yongfeng Sun, ${ }^{3}$ Jing Li, ${ }^{4}$ Yongning Lu, ${ }^{1}$ Xingang Li ${ }^{5,6}$
}

For numbered affiliations see end of article.

\section{Correspondence to} Professor Yongning Lu Union Hospital, Tongji Medical College, Huazhong University of Science and Technology, Wuhan 430022 China; 894367034@qq.com and Dr Xingang Li, Beijing Tiantan Hospital, Capital Medical University, No. 6 Tiantanxili, Dongcheng District, Beijing 100050, China; lxg198320022003@163.com

YG and MY contributed equally.

Received 29 September 2018 Revised 2 December 2018 Accepted 18 December 2018 Published Online First 19 January 2019

\section{EAHP Statement 4: Clinical} Pharmacy Services. EAHP Statement 5: Patient Safety and Quality Assurance. EAHP Statement 6: Education and Research.

\begin{abstract}
Objective Usage of tacrolimus is complicated by its narrow therapeutic index and wide between- and withinsubject pharmacokinetic variability. We aimed to obtain more information regarding the influence of various covariates on the disposition of tacrolimus in the early phase after cardiac transplantation using a population pharmacokinetic method, and provide information for the individualisation of drug dosing in the clinical setting. Methods Routine therapeutic drug monitoring concentrations (897 observations) were retrospectively collected from 146 hospitalised patients. One compartment model with first-order absorption (absorption rate constant $K_{a}$ was fixed as 4.48/ hour) was employed to establish the population pharmacokinetic model using a non-linear mixed-effects modelling approach. Various demographic parameters, postoperative day and concomitant medications influencing drug clearance and distribution volume were investigated in this study. Bootstrap and predictioncorrected visual predictive check were employed to validate the final model. With the goal of tacrolimus trough concentrations within the therapeutic window, simulation was performed.
\end{abstract}

Results Pharmacokinetic parameter population typical estimates for clearance (CL/F) and apparent distribution volume (V/F) were $14.23 \mathrm{~L} /$ hour and $760.80 \mathrm{~L}$, respectively. Postoperative day and co-administration of Wuzhi capsules were identified as important factors affecting CL/F. Total body weight was significantly associated with the V/F. Results of model evaluation indicated a good stable and precise performance of the final model. Based on the simulation results, a simpletouse dosage regimen table to guide clinicians with drug dosing was created.

Conclusion The final population model could provide information for the individualised dosing of tacrolimus for cardiac transplant recipients.

\section{INTRODUCTION}

Organ transplantation is the treatment of choice for patients with end-stage organ failure. Kidney and liver transplants accounted for $>90 \%$ of all large-organ transplants in China until 2011. ${ }^{1}$ In recent years, more and more heart transplants have been performed in China. ${ }^{1}$ Various pharmacokinetic (PK) studies of immunosuppressant agents, such as tacrolimus and ciclosporin, were mostly conducted among kidney or liver transplant patients. However, data involving heart transplantation was scarce. ${ }^{1}$ Heart failure is a systemic low perfusion syndrome resulting from impairment in the pumping function of the heart, resulting in a decrease in blood supply to various body organs with a potential to affect the PK of administered drugs. ${ }^{23}$ It has been reported that a 50\% reduction in systematic clearance of drugs (such as milrinone, carperitide, molsidomine, theophylline, ciclosporin and hydralazine) was observed in decompensated congestive heart failure (New York Heart Association (NYHA) III and IV). ${ }^{2}$ Guidelines for the use of tacrolimus in cardiac transplant recipients recommend that the target level of tacrolimus be achieved as soon as possible (within the first few days posttransplant). ${ }^{4}$ The reduced blood flow to the gastrointestinal tract, the peripheral tissues, as well as the liver and kidneys, may lead to a different PK profile of tacrolimus in a heart transplant recipient compared with a kidney or liver transplant patient.

Tacrolimus is a potent immunosuppressant drug primarily used to prevent and treat graft rejection after solid organ transplantation, and it exhibits higher patient and organ survival rates than ciclosporin. Moreover, tacrolimus leads to lower rejection rates and longer freedom from rejection. At present, when prioritising efficacy, tacrolimus is the first choice immunosuppressive drug for heart transplant recipients. ${ }^{5}$ Usage of tacrolimus is complicated by its narrow therapeutic index and wide between- and within-subject PK variability. ${ }^{6}$ Adequate drug exposure is needed for the prevention of rejection, but overexposures may increase the risk of toxicities, which affect longterm allograft and patient survival. Multiple factors may affect the dosing of tacrolimus. Regular therapeutic drug monitoring (TDM) is therefore essential for tacrolimus in clinical practice. With oral dosing, a steady-state blood concentration of tacrolimus is usually achieved within approximately 48 to 72 hours. Trough whole-blood levels for a heart transplant patient should be maintained between 15 to $20 \mathrm{ng} / \mathrm{mL}$ during the early postoperative period (days $0-60$ ), between 10 to $15 \mathrm{ng} / \mathrm{mL}$ during the intermediate period (2-6 months), and between 5 to $10 \mathrm{ng} / \mathrm{mL}$ after 6 to 9 months. ${ }^{4}$ Patient concentrations are often outside the target range.

Population PK is the study of sources and correlates of variability in drug exposure and response, and it describes the typical relationships between physiology and PK, the interindividual variability in these relationships, and their residual intraindividual variability. Knowledge of population kinetics can help to choose the initial drug dosage, and modify dosage appropriately in response to observed drug levels. Therefore, it is important to conduct a tacrolimus population PK study in cardiac transplant recipients. Knowledge of factors that affect PK can be derived from population PK studies. The non-linear mixed-effects modelling approach can offer the possibility to 


\begin{tabular}{|c|c|}
\hline Characteristic & Number or mean \pm SD (range or \%) \\
\hline Number of patients (male/female) & $146(116 / 30)$ \\
\hline Number of observations (male/female) & $891(731 / 160)$ \\
\hline Age (years) & $49.0 \pm 13.4(17-76)$ \\
\hline Total body weight (kg) & $66.0 \pm 14.8(29-124)$ \\
\hline Height $(\mathrm{cm})$ & $169.1 \pm 8.2(130-190)$ \\
\hline Serum creatinine $(\mu \mathrm{mol} / \mathrm{L})$ & $78.2 \pm 71.7(25.5-1100)$ \\
\hline Postoperative day & $12.0 \pm 11.9(1-96)$ \\
\hline Haemoglobin (g/L) & $112.0 \pm 18.5(14-168)$ \\
\hline Haematocrit (\%) & $34.0 \pm 5.3(11-66)$ \\
\hline Albumin (g/L) & $37.3 \pm 5.5(15-84)$ \\
\hline Total bilirubin ( $\mu \mathrm{mol} / \mathrm{L})$ & $16.4 \pm 33.2(25.6-102)$ \\
\hline Unconjugated bilirubin $(\mu \mathrm{mol} / \mathrm{L})$ & $8.9 \pm 18.0(1.4-210.7)$ \\
\hline Alkaline phosphatase (U/L) & $91.0 \pm 56.4(36-413)$ \\
\hline Alanine aminotransferase (U/L) & $35.0 \pm 65.7(6-968)$ \\
\hline Aspartate aminotransferase (U/L) & $23.0 \pm 62.2(9-545)$ \\
\hline$\gamma$-Glutamyl transpeptidase (U/L) & $78.0 \pm 156.4(11-1160)$ \\
\hline $\begin{array}{l}\text { Wuzhil capsule (+) } \\
\text { Prednisone }(+)\end{array}$ & $\begin{array}{l}53(36.3 \%) \\
146\end{array}$ \\
\hline Tacrolimus dose (mg/day) & $8.0 \pm 8.5(0.5-10)$ \\
\hline Drug concentrations $(\mu \mathrm{g} / \mathrm{mL})$ & $11.4 \pm 4.7(1.5-31.8)$ \\
\hline \multicolumn{2}{|l|}{ Main comorbidities } \\
\hline Infection & $45(30.8 \%)$ \\
\hline Graft failure & $30(20.5 \%)$ \\
\hline Renal dysfunction & $19(13.0 \%)$ \\
\hline Acute rejection & $16(11.0 \%)$ \\
\hline Hepatic dysfunction & $8(5.5 \%)$ \\
\hline
\end{tabular}

simultaneously characterise the PK parameters, and evaluate the variability of highly variable drugs with good precision and accuracy. Sparse data, such as routine TDM data, can be employed for this purpose.

In this study, TDM data of tacrolimus were collected from hospitalised heart transplant patients, using a population PK modelling method to evaluate the potential factors and to provide information for the individualisation of tacrolimus dosing.

\section{METHODS}

\section{Compliance with ethical standards}

All procedures followed were in accordance with the ethical standards of the responsible committee on human experimentation and with the Helsinki Declaration of 1964 and later versions. The study was conducted at Wuhan Union Hospital and the protocol was approved by the Research Ethics Committee of Wuhan Union Hospital (Ethical code: [2016]S249).

\section{Patient selection and data collection}

Routine TDM data (1-96 days post-operation) were retrospectively collected from 146 patients in Wuhan Union hospital from 1 January 2014 to 31 December 2015. The main information provided by the patient data is illustrated in table 1 . All patients were cardiac transplant recipients and were given tacrolimus capsules $(0.5 \mathrm{mg} / \mathrm{capsule}$ or $1.0 \mathrm{mg} / \mathrm{capsule}$, Prograf, AstellasPharma China, Inc) twice a day. The initial doses were calculated on a $0.10-0.15 \mathrm{mg} / \mathrm{kg} /$ day basis in two divided doses. Subsequent doses were adjusted based on clinical evidence of efficacy and toxicity, as well as tacrolimus TDM results ( 15 to $20 \mathrm{ng} / \mathrm{mL}$ ). We included adult heart transplant patients ( $\geq 17$ years) who were administered tacrolimus, corticosteroids and mycophenolate as the immunosuppressant therapy post-operation. Corticosteroids were started with intravenous administration $(2.0-2.5 \mathrm{mg} /$ kg every 8 hours) in the first 3 days and then switching to oral dosing; doses of the drug were gradually decreased over the days from transplantation. Mycophenolate was orally administered 24-48 hours after transplantation; the daily dose was $0.75-1 \mathrm{mg}$ every 12 hours in the first 2 weeks, and then reduced to $0.5 \mathrm{mg}$ every 12 hours.

Recipients who received combined lung-heart transplantation or were taking tacrolimus manufactured by other pharmaceutical companies were excluded. In addition, patients with incomplete medical record data were also excluded. In total, 10 patients were excluded from the study. All patients were hospitalised and treated under the supervision of medical and nursing staff.

The data were collected from medical records, including dose regimen of tacrolimus, date and time of blood sampling, drug concentrations, age (AGE), total body weight (BW), height (HT), serum creatinine ( $\mathrm{Scr}$ ), postoperative day (POD), sex (SEX), haemoglobin (HB), haematocrit (HCT), albumin (ALB), total bilirubin (TBIL), unconjugated bilirubin (IBIL), alkaline phosphatase (ALP), alanine aminotransferase (ALAT), aspartate aminotransferase (ASAT), $\gamma$-glutamyl transpeptidase (GGT) and concomitant medications of Wuzhi (WZ) capsules (multiple dosages of WZ capsules have been prescribed for the patients, and dosing for the heart transplant recipients ranged from 66 to $264 \mathrm{mg}$ per day, in two divided doses).

WZ capsules are a preparation of the ethanol herbal extract of Schisandra sphenanthera. The preparation was approved for clinical use in China for the protection of liver function after transplantation. Fundamental experiments have proven that the drug can affect the activity of the CYP3A enzyme, ${ }^{7}$ so it was included as a covariate screening in the study. As azoles were not regularly prescribed for the cardiac transplant recipients in Wuhan Union hospital, the co-administration effect of azoles (CYP3A inhibitors) were not investigated in this study. We reconfirmed the information before model development.

\section{Tacrolimus determination}

Tacrolimus concentration measurement was determined by Viva-E Drug Testing System (Seimens Healthcare, Erlangen, Germany) ${ }^{8}$ with enzyme multiplied immunoassay technique. The lower limit of quantitation of the assay was $2 \mathrm{ng} / \mathrm{mL}$, ranging from 2 to $30 \mathrm{ng} / \mathrm{mL}$.

\section{Population PK model}

Population PK analysis was carried out using the Phoenix NLME 8.0 programme (Certara, St Louis, Missouri, USA). A onecompartment with first-order absorption model was employed. The First-order Conditional Estimation with Extended Least Squares (FOCE-ELS: this method is essentially equivalent to the NONMEM FOCE methodology with interaction) method was used throughout the process of model building. In order to avoid the effect of fluctuation on the stability of the model, ${ }^{9}$ the value of $\mathrm{K}_{2}$ (absorption constant) was fixed as 4.48/hour at the stage of stacking the covariates. ${ }^{10}$

In the first step, an exploratory analysis of the mean, SD and coefficient of variation of all candidate variables was performed. Graphical and linear regressions were used to test the relationship of PK variables versus covariates. Additionally, the interrelation between variables was also analysed in order to eliminate components from the candidate predictor relationships. In the process of model development, continuous covariates (POD, AGE, BW, HT, SCR, HB, HCT, ALB, TBIL, IBIL, ALP, ALAT, ASAT, GGT) were evaluated using the following equations: 


$$
P_{i}=t v P \times\left(\operatorname{Cov} / \operatorname{Cov} v_{\text {median }}\right)^{\theta}
$$

where $P_{i}$ represents the individual parameter estimate of the $i$ th subject, $t v P$ represents the typical value of this parameter, $\mathrm{Cov}$ and $\mathrm{Cov}_{\text {median }}$ are the covariate and the median of covariate, respectively, and $\theta$ is the corresponding coefficient. Preliminary PK analysis indicated that the proportional model was suitable for the description of residual error.

$$
C_{i}=C \times(1+\varepsilon)
$$

where $C_{i}$, and $C$ are individual observations and predictions, respectively. $E$ accounts for the proportional error of predictions, which is normally distributed with mean zero and variances of $\sigma^{2}$. Categorical covariates (SEX: male $=1$ and female $=2$; WZ capsules: $c 0$-administration $=1$ and none $=0$ ) were incorporated using indicator variables.

Initially, a structure model was formed without any covariates. The candidate covariates were then added to the structure model to identify its significance in turn. If objective function value (OFV) of the model reduced more than $3.84(\mathrm{p}<0.05, d f=1)$, the covariate was considered to be retained in the model. After the stage of forward selection, covariates need to be re-evaluated by improving the test level $(\mathrm{p}<0.001, d f=1)$ in the stage of backward elimination. Each covariate was independently removed from the model one at a time to identify its relevance. An increase in the $\Delta \mathrm{OFV}$ of over 10.83 was required for confirmation. When two or more covariates were found to significantly improve the model, the covariate causing the largest reduction was left in the final model.

\section{Model evaluation}

A visual evaluation method was carried out by inspection of the scatter plots of drug concentrations versus population prediction (PRED) and individual predicted (IPRED) concentrations. The relative prediction errors were graphically described by conditional weighted residuals (CWRES) plotted against PRED and time. Additionally, the Normal Quantile-Quantile (QQ) plot was also used to evaluate how well the distribution of CWRES matched a standard normal distribution.

To evaluate the stable and predictive performance of the final model, a non-parametric bootstrap method and a visual prediction-corrected predictive check (pcVPC) was carried out after the model building. The bootstrap method involves repeated random re-sampling with replacement from the original dataset for 1000 times. More than 800 successful re-samplings were required for the stability evaluation of the final model. Additionally, the values of estimated parameters, such as the median and $95 \% \mathrm{CI}$, from the bootstrap procedure were compared with those estimated from the original dataset. No significant difference was observed, indicating a reliable final model. The pcVPC used 1000 times Monte Carlo simulation to generate concentration-time profiles. In a pcVPC, the variability coming from binning across independent variables is removed by normalising the observed and simulated dependent variables based on the typical population prediction for the median independent variable in the bin. The pcVPC has an enhanced ability to diagnose model misspecification, especially with respect to random effects models in a range of situations. The observed concentration-time data were graphically superimposed on the median values, and the 5 th and 95 th percentiles of the simulated concentration-time profiles. The model was deemed precise if the observed concentration data were approximately distributed within the 5 th and 95 th prediction interval.

\section{Simulation}

This study aimed to provide dosing guidance for the use of tacrolimus in cardiac transplant recipients. Trough wholeblood levels for a heart transplant patient should be maintained between 15 to $20 \mathrm{ng} / \mathrm{mL}$ during the early postoperative period (0-2 months), and between 10 to $15 \mathrm{ng} / \mathrm{mL}$ during the intermediate period (2-6 months). After the final model was developed, tacrolimus concentrations at different times were calculated based on the relationship between PK parameters and the covariates. Patients were divided into different subgroups according to covariates significantly associated with PK. Simulations were conducted using the Phoenix NLME software to find the optimal individualised dosing regimen for different subgroups of patients. Simulation was performed to ensure that $>90 \%$ of the trough concentrations were within target concentration during the therapy. A simple to use dosage regimen table was derived based on the results of simulations.

\section{RESULTS}

\section{Model development}

A one-compartment with first-order absorption model best fitted the observed concentrations. The parameter estimates of CL and $\mathrm{V} / \mathrm{F}$ were $14.01 \mathrm{~L} /$ hour and $869.61 \mathrm{~L}$, respectively. When adding the candidate covariates separately to the structure model, CL was markedly affected by POD and the co-administration of WZ; V/F was significantly influenced by BW. After forward selection and backward elimination of all the covariates, the final model presented as follows:

$$
\begin{gathered}
C L / F(L / h)=14.23 \times(P O D / 12)^{-0.12} \times \exp (0.39 \times(W Z=1)) \times \exp (n C L) \\
V / F(L)=846.91 \times(B W / 66)^{2.06} \times \exp (n V)
\end{gathered}
$$

where in equation 3, POD was calculated by days; when patients were co-administered WZ, the value of item ' $\exp (0.39 \times(\mathrm{WZ}=1))$ ' is 'exp(0.39)', otherwise the values 1 . 12 and 66 stand for the median value of POD and BW, respectively. Parameter estimates for the final model are summarised in table 2. A good estimation of all the model parameters was obtained (relative standard error (RSE) <30.0\%).

\section{Goodness-of-fit}

Goodness-of-fit plots were used to assess the reliability of both the structural model and the final pharmacokinetic model. These plots included five scatter plots: observations against PRED, observations against IPRED, CWRES versus time, CWRES versus PRED, and Normal QQ plots (figure 1). Goodness-of-fit plots did not show systematic bias for both structural and final pharmacokinetic model predictions. A significant improvement in the predictive performance of the final model (figure 1A'-E') was achieved as compared with the basic one (figure 1A-E).

\section{Model evaluation}

The validation of the final model by bootstrapping and pcVPC also indicated satisfactory results. A total of 966 re-samplings of the original dataset were successfully performed in the process of bootstrap evaluation, indicating a qualified stability for the final model. Table 2 also summarises the parameter estimates for the final model and bootstrap replicates; the comparable data values indicate a good prediction of the final model. The distribution of the 1000 simulated concentration-time curves and the comparison with the observations in the original dataset are shown in figure 2. Most determined concentrations were within the $90 \%$ prediction interval of model-predicted concentrations, 
Table 2 Final model characteristics and bootstrap results

\begin{tabular}{|c|c|c|c|c|c|}
\hline \multirow[b]{2}{*}{ Parameters } & \multicolumn{3}{|l|}{ Final model } & \multicolumn{2}{|c|}{ Bootstrap (1000) } \\
\hline & Estimate (RSE \%) & IIV (CV\%) & Shrinkage & Median & $95 \% \mathrm{Cl}$ \\
\hline \\
\hline \multicolumn{6}{|c|}{$\begin{array}{l}C L / F(L / h)=t v C L \times\left(P O D / P O D_{\text {median }}\right)^{\theta_{1}} \times \exp \left(\theta_{2} \times(W Z=1)\right) \times \exp (n C L) \\
\text { Volume of distribution: }\end{array}$} \\
\hline \multicolumn{6}{|c|}{$V / F(L)=t v V \times\left(B W / B W_{\text {median }}\right)^{\theta_{3}} \times \exp (n V)$} \\
\hline $\operatorname{tv} C L / F(L / h)$ & $14.23(5.39)$ & 38.89 & 0.12 & 14.14 & 12.29 to 16.08 \\
\hline$t V V / F(L)$ & $846.91(9.09)$ & 85.95 & 0.19 & 847.87 & 698.55 to 1006.73 \\
\hline$\theta_{1}$ & $-0.12(20.02)$ & - & - & -0.12 & -0.19 to -0.02 \\
\hline e & $0.39(19.00)$ & - & - & 0.39 & 0.24 to 0.52 \\
\hline$\theta_{3}$ & $2.06(22.33)$ & - & - & 2.04 & 1.33 to 2.81 \\
\hline \multicolumn{6}{|c|}{ Residual error model (proportional error, CV\%) } \\
\hline Proportional & $0.30(3.02)$ & - & 0.13 & 0.30 & 0.27 to 0.32 \\
\hline
\end{tabular}

$\mathrm{CV}$, coefficient of variation; IIV, inter-individual variability; RSE, relative standard error.

suggesting the precise performance of the final model. However, pcVPC plots showed that the median of the observed and predicted concentration was not in agreement in the large POD. In this study, most of the data were in the POD $<40$ days, and we only obtained limited pharmacokinetic data in the large POD. We tried our best to optimise the final model and this was the best one we could get. This deviation may come from a random error.

\section{Simulation}

A total of three covariates (POD, WZ and BW) were incorporated into the final model, and 12 subgroups were obtained according to the incorporated covariates. The range of POD during simulation was restricted to the ranges observed in the modelling data (1-96 days) to prevent distortion of results. The values of $\mathrm{CL} / \mathrm{F}$ and $\mathrm{V} / \mathrm{F}$ were calculated using equations 3 and 4 , respectively ( $\mathrm{K}_{\mathrm{a}}$ were fixed as $4.48 /$ hour). With the goal of having tacrolimus trough concentrations maintained within $15-20 \mathrm{ng} / \mathrm{mL}$ (POD =0-2 months) and 10-15 ng/mL (POD =2-6 months), the optimal dosage regimens for different subgroups and percentage of trough concentrations within target concentration are listed in table 3 . The results of this simulation were only used for patients whose POD were within 1-96 days. Simulation results showed that as POD increased, oral doses needed to be decreased as well to maintain optimal exposures. However, splitting the tacrolimus capsules is not convenient for patients. Based on the simulation results, we found that adjusting the calculated dosage according to the drug specification was acceptable (eg, $3.5 \mathrm{mg}$ instead of $3.6 \mathrm{mg}, 2.0 \mathrm{mg}$ instead of $1.8 \mathrm{mg}$ or $2.2 \mathrm{mg}$, $2.5 \mathrm{mg}$ instead of $2.3 \mathrm{mg}$ ).

\section{DISCUSSION}

In this study, routine clinical PK data were retrospectively collected from 146 cardiac transplant patients in Wuhan Union Hospital to investigate potential covariates which may affect the PK parameters. A population PK model conducted on Chinese heart transplant patients has been built through the Phoenix NLME programme. Typical value of $\mathrm{CL} / \mathrm{F}$ was $14.23 \mathrm{~L} /$ hour $\left(t v C L / F / B W_{\text {median }}=0.22 \mathrm{~L} /\right.$ hour $/ \mathrm{kg}$ ), which is relatively consistent with the estimated range $(0.19-0.23 \mathrm{~L} /$ hour $/ \mathrm{kg})$ published in the literature. ${ }^{11}$ Since $\mathrm{K}_{\mathrm{a}}$ was fixed, sensitivity analysis was performed to assess its impact on the $\mathrm{CL} / \mathrm{F}$ and V/F. Because most of sampling points were in the elimination phase (trough concentration), $\mathrm{K}_{\mathrm{a}}$ did not influence the $\mathrm{CL} / \mathrm{F}$ and V/F significantly. In contrast to the published population PK investigations in China, tacrolimus PK in cardiac transplant recipients (0.22L/hour/kg; POD: $12.0 \pm 11.9$ days) showed a decreased clearance compared with healthy volunteers (CL: $0.52 \mathrm{~L} / \mathrm{hour} / \mathrm{kg}$; POD: $6.24 \pm 1.19$ days). When we set the value of POD to $6-7$, the
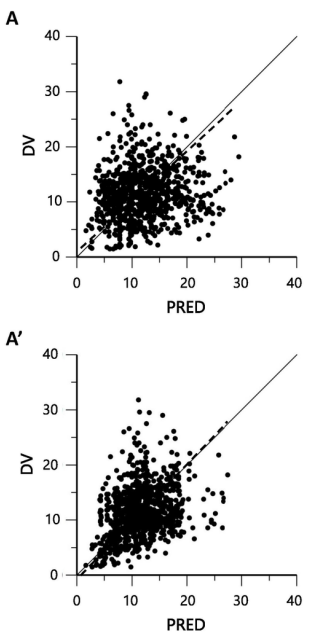

B
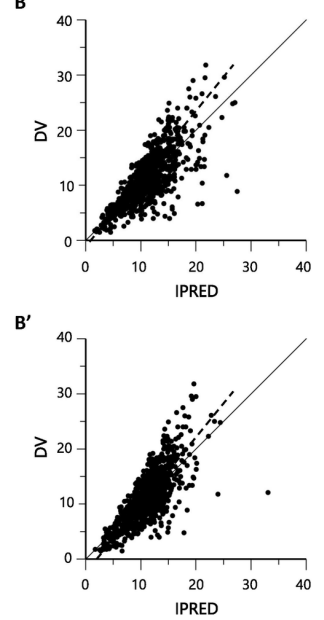

C

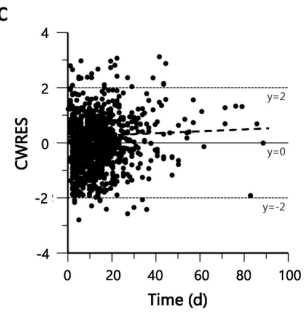

$C^{\prime}$

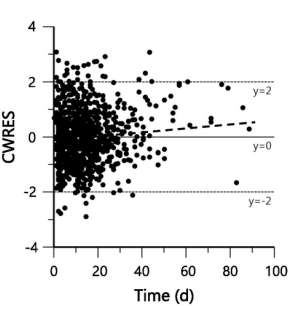

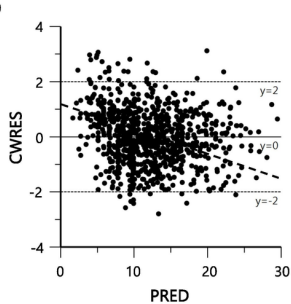

$D^{\prime}$

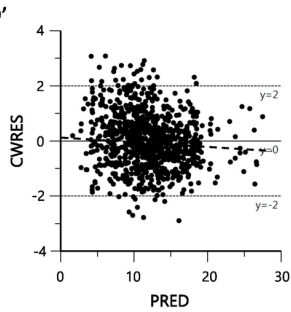

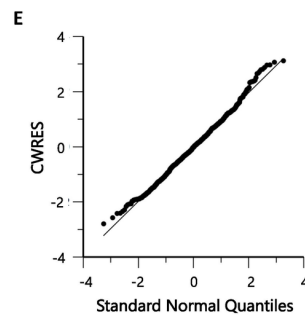

$E^{\prime}$

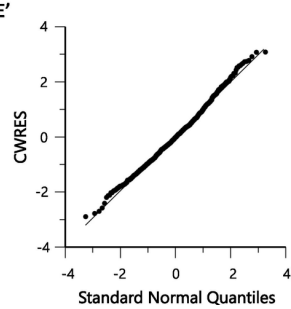

Figure 1 Diagnostic scatter plots of the final model. A: dependent value (DV, observed concentrations) versus population predicted concentrations (PRED); B: DV versus individual population predicted concentrations (IPRED); C: conditional weighted residuals (CWRES) versus time; D: CWRES versus PRED; E: Normal QQ plot of CWRES. The dashed lines represent trend lines. 

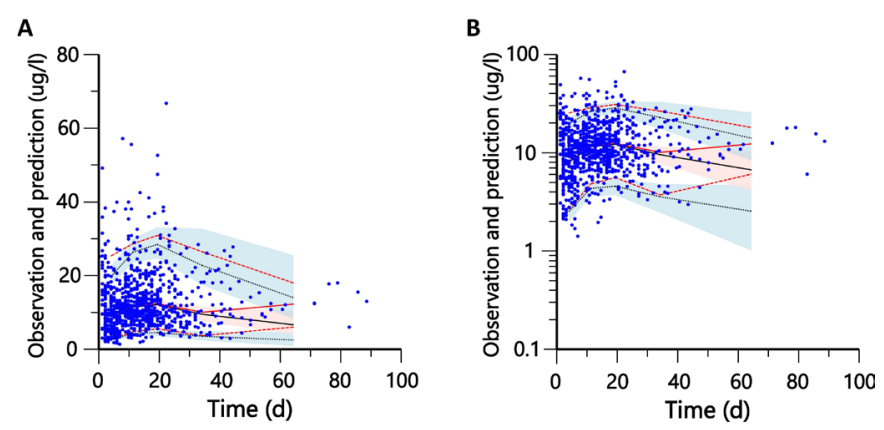

Figure 2 Prediction-corrected visual predictive check (pcVPC) obtained from 1000 simulations of the database. A: ordinary coordinate. $\mathrm{B}$ : logarithmic coordinates. The dots are the prediction-corrected drug concentrations. The black solid line represents predicted 50th percentile and the black dotted lines mean the 5th and 95th percentiles (shadow means $95 \%$ confidence band), indicating the areas between the 5 th and 95th percentiles representing the $90 \%$ prediction interval. Similarly, the red solid line means the observed 50th percentile and the red dashed lines are the 5th and 95th percentiles of observations.

value of tacrolimus clearance calculated by our model was about $0.23 \mathrm{~L} /$ hour $/ \mathrm{kg}$, which decreased by about $56 \%$ compared with the healthy volunteers. ${ }^{12}$ This value is similar to clearance in liver transplant patients (CL: 0.26L/hour/kg; POD: 7.72 44.19 days; age: $58.4 \pm 11.6$ years). ${ }^{12}$ In the unstable clinical phase, whole blood clearance of tacrolimus and its metabolites is influenced by several adverse factors, among which reduced blood flow to various organs, severe cholestasis, anaemia and hypoalbuminaemia may all substantially alter the clearance. Low blood perfusion in liver and cholestasis (reflecting hepatic dysfunction) decrease the metabolism and transport of tacrolimus into the bile, resulting in a reduced clearance of tacrolimus. ${ }^{5}$ Rower et al constructed a population PK model that describes tacrolimus concentrations in paediatric patients receiving a heart transplant. Due to the differences between adults and children, PK parameters, CL/F (9.5 L/h) and V $(233 \mathrm{~L})$ were significantly lower than those in this model. ${ }^{13}$

In this study, tacrolimus clearance was found to vary with POD and the co-administration of WZ capsules; distribution volume was significantly influenced by BW. POD is a significant covariate with high recognition among various studies. ${ }^{14-16}$ Tacrolimus is a fat-soluble drug, which binds strongly to erythrocytes and plasma proteins. Recovery of gastrointestinal function and increased intake of food can alter tacrolimus bioavailability. ${ }^{15}$ The combined effects lead to an increase in $\mathrm{CL} / \mathrm{F}$ as POD increases. Recovery of the gastrointestinal function may play a leading role in the absorption of tacrolimus, which leads to an increase in the clearance of tacrolimus. TDM data included

Table 3 Dosing regimens (twice a day, $\mu \mathrm{g}$ ) for cardiac transplant recipients based on the $\mathrm{POD}, \mathrm{BW}$ and drug combination

\begin{tabular}{llll}
\hline \multicolumn{4}{l}{ POD $=0$-60 days (trough concentration within 15-20 ng/mL) } \\
\hline Drug combination & $\mathrm{BW}<60 \mathrm{~kg}$ & $\mathrm{BW}=60-80 \mathrm{~kg}$ & $\mathrm{BW}>80 \mathrm{~kg}$ \\
Without WZ & $2500(93.3)^{*}$ & $2500(92.2)^{*}$ & $2500(90.2)^{*}$ \\
With WZ & $3500(96.8)^{*}$ & $3500(94.1)^{*}$ & $3600(91.3)^{*}$ \\
\hline POD=61-96 days (trough concentration within 10-15 ng/mL) \\
\hline Drug combination & $\mathrm{BW}<60 \mathrm{~kg}$ & $\mathrm{BW}=60-80 \mathrm{~kg}$ & $\mathrm{BW}>80 \mathrm{~kg}$ \\
Without WZ & $1800(91.7)^{*}$ & $2200(92.1)^{*}$ & $2300(90.2)^{*}$ \\
With WZ & $2500(95.6)^{*}$ & $2500(93.5)^{*}$ & $2500(90.4)^{*}$
\end{tabular}

*Percentage of trough concentrations within target concentrations during therapy. BW, body wieght; POD, postoperative day; WZ, Wuzhi. in this study ranged from 1 to 96 days post-transplantation, so that POD played an important role in the variance of $\mathrm{CL}$ between patients. In Rower's model, data were collected within the first 6 weeks after heart transplantation. That study identified age, creatinine clearance and fluconazole use as patientspecific factors that have an impact on tacrolimus PK.

The Chinese medicine WZ capsules are a preparation of ethanol herbal extract of Schisandra sphenanthera. The main active ingredients include schisandrin, schizandrol B, schisantherin A, schisanhenol and dexyschizandrin. ${ }^{17}$ Several clinical studies reported in China indicated that the co-administration of WZ capsules could significantly increase peak drug concentration and AUC of tacrolimus in kidney or liver transplant patients. ${ }^{18-20}$ In addition, several fundamental experiments on WZ capsules have been conducted in China. Some of the results showed that tacrolimus blood concentration increased when co-administered with single or multiple doses of WZ capsules. ${ }^{717}$ However, there are also some publications that reported that Schisandra lignan extract induced both CYP3A and CYP2C expression through activation of the orphan nuclear receptor pregnane $\mathrm{X}$ receptor, probably by exerting a biphasic effect (short-term inhibition and long-term induction) on regulating CYP3A expression and activity, which was also observed with St John's wort. ${ }^{21} 22$ Recently, long-term administration of the Schisandra lignan extract was found to induce both intestinal and hepatic CYP3A protein expression, suggesting that WZ capsules exhibited a mechanism-based induction toward CYP3A. ${ }^{23}$ Tacrolimus is primarily metabolised in the liver or small intestine by CYP3A. ${ }^{10}$ The induction effect of Schisandra lignan extract on CYP3A protein expression may partly explain the tacrolimus clearance increase observed in heart transplant patients when co-administered with WZ capsules in our study.

As we know, tacrolimus metabolism depends on CYP3A5, and CYP3A5 may be the most important genetic contributor to interindividual and interracial differences in CYP3A-dependent drug clearance and in responses to many medicines. Single nucleotide polymorphisms in CYP3A5*3 causes alternative splicing and protein truncation, resulting in the absence of CYP3A5 from tissues of some people. ${ }^{24}$ Several studies have reported the association between the CYP3A5*3 polymorphism and tacrolimus PKs. ${ }^{25-27}$ Patients with the CYP3A5*3 carriers require less tacrolimus to obtain target pre-dose concentrations compared with patients with the CYP3A5*1/*1 genotype. Except for CYP3A $5 * 3$, the allele CYP3A4*18B was also significantly associated with dose-adjusted tacrolimus blood trough concentrations and had a strong time-genotype interaction with tacrolimus PK. Thus, genotyping of the CYP $3 A 4$ and CYP $3 A 5$ genes should be considered with respect to determining tacrolimus dose regimens during the post-transplantation period. ${ }^{28}$

Tacrolimus is a fat-soluble drug and can easily distribute to the peripheral compartment. ${ }^{14}$ Therefore, the factor of body weight can impose a significant impact on V/F. Many studies have indicated that haematocrit was an important variable affecting the $\mathrm{V} / \mathrm{F}$ of tacrolimus, as $85-95 \%$ of tacrolimus was found within erythrocytes in blood. ${ }^{515} 16$ However, in this study, the variation of HCT and ALB among patients was not that wide (table 1), so that the significance of the covariates has not been identified.

Because of the incomplete records of patient information, genetic factors were not incorporated into this study. As a widely recognised factor, genetic mutations of CYP3A have been demonstrated to affect the dosage of tacrolimus in kidney or liver transplant patients. ${ }^{1}$ More recently, investigators in the USA found that the combined CYP3A genotype was associated with tacrolimus drug disposition in adult transplant 
What this paper adds

What is already known on this subject

- Due to a narrow therapeutic index and wide between- and within-subject pharmacokinetic variability of tacrolimus, routine therapeutic drug monitoring is necessary for patients.

- The postoperative day is a well-known covariate affecting tacrolimus clearance.

\section{What this study adds}

- Tacrolimus pharmacokinetics in Chinese cardiac transplant recipients showed a decreased clearance compared with healthy volunteers.

- The covariates postoperative day, Wuzhi capsules and body weight were identified as the important factors affecting tacrolimus clearance and distribution volume in heart transplant patients.

patients, but the effect was largely driven by CYP3A $5 * 3 .{ }^{29}$ Genetic polymorphisms of metabolising enzymes and transport proteins should be investigated for the individualisation of tacrolimus dosing for cardiac transplant patients in further prospective studies.

In summary, the well-known covariates POD, WZ capsules and BW were successfully identified as the important factors affecting tacrolimus $\mathrm{CL} / \mathrm{F}$ and $\mathrm{V} / \mathrm{F}$ in heart transplant patients. This model was stable and precise, and simulation was performed based on the final PK model. The final model could provide information for the individualised dosing of tacrolimus.

\section{Limitations}

Several limitations should be addressed. (1) Cardiotoxicity is found among people who take tacrolimus, (taking the drug for $<1$ month). The tacrolimus pre-dose concentration is associated with increased left ventricular wall thickness in paediatric liver transplant recipients. Systolic and diastolic hypertension is associated with tacrolimus pre-dose concentration, ${ }^{30}$ therefore patients with a higher target tacrolimus pre-dose concentration require a more intensive blood pressure surveillance (ambulatory blood pressure measurements). However, information about the ambulatory blood pressure measurements was not obtained in this study. (2) The fact that only pre-dose concentrations rather than PK profiles were used to build the model is an obvious limitation of this study. Design of optimal sampling times for PK analysis is very important. However, this is a retrospective study and we cannot design the sampling time points. We are planning to collect data for an external validation. However, it will take a long time to collect enough data to validate our final model. (3) WZ capsule administration was included as a covariate with the hypothesis that the drug can alter CYP3A activity; however, the metabolite(s) of tacrolimus via the CYP3A pathway were not quantitated.

\footnotetext{
Author affiliations

'Department of Pharmacy, Union Hospital, Tongji Medical College, Huazhong University of Science and Technology, Wuhan, China

${ }^{2}$ Department of Anesthesiology, Tianjin Eye Hospital, Tianjin, China

${ }^{3}$ Department of Cardiovascular Surgery, Union Hospital, Tongji Medical College, Huazhong University of Science and Technology, Wuhan, China

${ }^{4}$ Department of Pharmacy, Children's Hospital of Fudan University, Shanghai, China

${ }^{5}$ Department of Pharmacy, Beijing Tiantan Hospital, Capital Medical University, Beijing, China

${ }^{6}$ Precision Medicine Center for Neurological Disorders, Beijing Tiantan Hospital, Capital Medical University, Beijing, China
}

Acknowledgements Professor Shusen Sun, Western New England University, Springfield, Massachusetts, provided a lot of help in the article writing process. We gratefully acknowledge his kindness.

Contributors $Y L$ and $X L$ conceived and designed this study. $Y G, M Y$ and $X L$ performed the analysis. YG and XL wrote the manuscript. YL, YS, and JL supervised the quality of the study. All authors read and approved the final manuscript.

Funding This project was supported by a grant from Beijing Municipal Administration of Hospitals' Youth Programme (QML20170506) and Young Scientist Program (YSP201703)

Competing interests None declared.

Patient consent Next of kin consent obtained.

Provenance and peer review Not commissioned; internally peer reviewed.

ORCID iD

Xingang Li http://orcid.org/0000-0001-6726-9571

\section{REFERENCES}

1 Hu XJ, Dong NG, Liu JP, et al. Status on heart transplantation in China. Chin Med J 2015; 128:3238-42.

2 Ogawa R, Stachnik JM, Echizen H. Clinical pharmacokinetics of drugs in patients with heart failure: an update (part 2, drugs administered orally). Clin Pharmacokinet 2014;53:1083-114.

3 Yancy CW, Jessup M, Bozkurt B, et al. ACC/AHA/HFSA focused update on new pharmacological therapy for heart failure: an update of the 2013 ACCF/AHA guideline for the management of heart failure: a report of the American College of Cardiology/ American Heart Association Task Force on Clinical Practice Guidelines and the Heart Failure Society of America. J Am Coll Cardiol 2016;2016:1476-88.

4 Taylor DO, Barr ML, Meiser BM, et al. Suggested guidelines for the use of tacrolimus in cardiac transplant recipients. J Heart Lung Transplant 2001;20:734-8.

5 Sikma MA, van Maarseveen EM, van de Graaf EA, et al. Pharmacokinetics and toxicity of tacrolimus early after heart and lung transplantation. Am J Transplant 2015;15:2301-13.

6 Bergmann TK, Hennig S, Barraclough KA, et al. Population pharmacokinetics of tacrolimus in adult kidney transplant patients: impact of CYP3A5 genotype on starting dose. Ther Drug Monit 2014;36:62-70.

7 Qin $X L$, Chen $X$, Wang Y, et al. In vivo to in vitro effects of six bioactive lignans of Wuzhi tablet (Schisandra sphenanthera extract) on the CYP3A/P-glycoproteinmediated absorption and metabolism of tacrolimus. Drug Metab Dispos 2014;42:193-9.

8 Chung HS, Lee ST, Lee SY. [Evaluation of Viva-E drug testing system]. Korean J Lab Med 2007;27:330-7.

9 Gong Y, Chen Y, Li Q, et al. Population pharmacokinetic analysis of digoxin in Chinese neonates and infants. J Pharmacol Sci 2014;125:142-9.

10 Zhang JJ, Zhang $\mathrm{H}$, Ding XL, et al. Effect of the P450 oxidoreductase 28 polymorphism on the pharmacokinetics of tacrolimus in Chinese healthy male volunteers. Eur J Clin Pharmacol 2013;69:807-12.

11 McCormack PL, Keating GM. Tacrolimus: in heart transplant recipients. Drugs 2006;66:2269-79.

12 Lu YX, Su QH, Wu KH, et al. A population pharmacokinetic study of tacrolimus in healthy Chinese volunteers and liver transplant patients. Acta Pharmacol Sin 2015:36:281-8.

13 Rower JE, Stockmann C, Linakis MW, et al. Predicting tacrolimus concentrations in children receiving a heart transplant using a population pharmacokinetic model. BMJ Paediatr Open 2017;1:e000147.

14 Guy-Viterbo V, Scohy A, Verbeeck RK, et al. Population pharmacokinetic analysis of tacrolimus in the first year after pediatric liver transplantation. Eur J Clin Pharmacol 2013;69:1533-42.

15 Zhao CY, Jiao Z, Mao JJ, et al. External evaluation of published population pharmacokinetic models of tacrolimus in adult renal transplant recipients. Br J Clin Pharmacol 2016;81:891-907.

16 Zhao W, Fakhoury M, Baudouin V, et al. Population pharmacokinetics and pharmacogenetics of once daily prolonged-release formulation of tacrolimus in pediatric and adolescent kidney transplant recipients. Eur J Clin Pharmacol 2013;69:189-95.

17 Wei H, Tao X, Di P, et al. Effects of traditional Chinese medicine Wuzhi capsule on pharmacokinetics of tacrolimus in rats. Drug Metab Dispos 2013;41:1398-403.

18 Jiang W, Wang X, Xu X, et al. Effect of Schisandra sphenanthera extract on the concentration of tacrolimus in the blood of liver transplant patients. Int I Clin Pharmacol Ther 2010;48:224-9.

19 Xin HW, Li Q, Wu XC, Xc W, et al. Effects of Schisandra sphenanthera extract on the blood concentration of tacrolimus in renal transplant recipients. Eur I Clin Pharmacol 2011;67:1309-11.

20 Xin HW, Wu XC, Li Q, et al. Effects of Schisandra sphenanthera extract on the pharmacokinetics of tacrolimus in healthy volunteers. Br J Clin Pharmacol 2007;64:469-75. 


\section{Original research}

21 Rengelshausen J, Banfield M, Riedel KD, et al. Opposite effects of short-term and long-term St John's wort intake on voriconazole pharmacokinetics. Clin Pharmacol Ther 2005;78:25-33.

22 Xie HG, Kim RB. St John's wort-associated drug interactions: short-term inhibition and long-term induction? Clin Pharmacol Ther 2005;78:19-24.

23 Lai L, Hao H, Wang Q, et al. Effects of short-term and long-term pretreatment of Schisandra lignans on regulating hepatic and intestinal CYP3A in rats. Drug Metab Dispos 2009;37:2399-407.

24 Kuehl P, Zhang J, Lin Y, et al. Sequence diversity in CYP3A promoters and characterization of the genetic basis of polymorphic CYP3A5 expression. Nat Genet 2001;27:383-91.

25 Gijsen VM, van Schaik RH, Elens L, et al. CYP3A4*22 and CYP3A combined genotypes both correlate with tacrolimus disposition in pediatric heart transplant recipients. Pharmacogenomics 2013;14:1027-36.
26 Hesselink DA, van Schaik RH, van der Heiden IP, et al. Genetic polymorphisms of the CYP3A4, CYP3A5, and MDR-1 genes and pharmacokinetics of the calcineurin inhibitors cyclosporine and tacrolimus. Clin Pharmacol Ther 2003:74:245-54.

27 Prytuła AA, Cransberg K, Bouts AH, et al. The effect of weight and CYP3A5 genotype on the population pharmacokinetics of tacrolimus in stable paediatric renal transplant recipients. Clin Pharmacokinet 2016;55:1129-43.

28 Liu F, Ou YM, Yu AR, et al. Long-term influence of CYP3A5, CYP3A4, ABCB1, and NR1I2 polymorphisms on tacrolimus concentration in Chinese renal transplant recipients. Genet Test Mol Biomarkers 2017;21:663-73.

29 Deininger KM, Vu A, Page RL, et al. CYP3A pharmacogenetics and tacrolimus disposition in adult heart transplant recipients. Clin Transplant 2016;30:1074-81.

30 Prytula A, Vandekerckhove K, Raes A, et al. Tacrolimus predose concentration is associated with hypertension in pediatric liver transplant recipients. J Pediatr Gastroenterol Nutr 2016:63:616-23. 